\title{
Relato de caso de Lagochilascariose humana procedente do Estado do Pará, Brasil
}

\author{
Report a case of human lagochilascariasis coming \\ from the Pará State, Brazil \\ Miguel Alípio Vieira, Jayrson Araújo de Oliveira, Lisiane Seguti Ferreira, \\ Viviane de Oliveira e Carlos Augusto Lopes Barbosa
}

Resumo Mais um caso de lagochilascariose em criança procedente do Município de Xinguara PA. No Hospital das Clínicas da UFG, foi feita drenagem do abscesso localizado na região cervical direita, constatando-se a presença de ovos e vermes adultos de Lagochilascaris minor. Instituída terapia com albendazol $400 \mathrm{mg} /$ dia (durante 30 dias) e antibioticoterapia, houve regressão do quadro clinico.

Palavras-chaves: Lagochilascariose. Albendazol. Novo caso. Brasil.

Summary $A$ new case of lagochilascariasis is reported in a child from Xinguara PA, Brazil. The patient had an abscess in the right cervical region, which was drained at the Clinical Hospital of UFG. Eggs and adult stages of Lagochilascaris minor were found in the secretion of the abscess. Treatment with albendazol, at a dosage of $400 \mathrm{mg} /$ day for 30 days, associated with antibiotics promoted regression of the lesion.

Key-words: Lagochilascariose. Albendazol. New case. Brazil.

O Lagochilascaris minor Leiper, 190920, dentre as cinco espécies descritas neste gênero é a mais importante, uma vez que tem sido encontrado mais de 100 casos de parasitismo humano ${ }^{17} 1829$. A doença é caracterizada pela presença de abcessos supurados, especialmente localizados ao nível do pescoço, ouvido médio e região mastóide, apesar de que, tem sido reportado seu encontro em outros locais, tais como: sistema nervoso central ${ }^{11} 152428333645$; pulmões ${ }^{22} 2344$; osso sacro $^{21}$, alvéolo dentário ${ }^{37}$, seios paranasais ${ }^{44}$, globo ocular ${ }^{33}$ e região temporo-parieto-occipital ${ }^{2}$.
Dos abcessos, frequentemente, observa-se a eliminação de diferentes estágios evolutivos do parasito junto com secreções através de fístula14571213161922 252633 34353940 .

O Brasil lidera a casuística mundial e a maioria dos casos humanos tem distribuição geográfica restrita à amazônia legal, especialmente o Estado do Pará.

Apresentamos mais um caso de lagochilascariose humano procedente do município de Xinguara, sul do Pará.

\footnotetext{
Trabalho realizado no Hospital das Clínicas da Universidade Federal de Goiás e Instituto de Patologia Tropical e Saúde Pública da Universidade Federal de Goiás.

Endereço para correspondência: Instituto de Patologia Tropical e Saúde Púiblica/UFG. Rua Delenda Rezende de Melo s/n, Setor Universitário, 74605-005 Goiânia, GO,

Telefax: 5562 261-6497

E-mail: jayrson@ipe.ufg.br

Recebidopara publicação em 7/1/99.
} 


\section{RELATO DO CASO}

Criança de 8 anos de idade, do sexo masculino (J.C.M.), procedente da zona rural do município de Xinguara, PA.

Os sintomas iniciaram há cerca de três anos, com quadro clínico de otite de repetição com otorréia. Durante este período foi submetido a diversos tratamentos clínicos e duas intervenções cirúrgicas, sendo uma delas a mastoidectomia radical, sem melhora completa.

Em 6/12/95 foi admitido no Hospital das Clínicas da Universidade Federal de Goiás apresentando febre, emagrecimento acentuado, astenia, queda do estado geral, além de tumoração com sinais flogísticos na região cervical e otorréia fética abundante. O hemograma apresentou valores normais, tanto a série vermelha como a série branca.

Uma semana após internação foi feita drenagem do abcesso infrauricular com eliminação de grande quantidade de vermes. No exame bacteriológico da secreção do abcesso auricular, foram isolados cocos gram positivos.
Tomografia computadorizada mostrou múltiplas áreas líticas no temporal direito estendendo-se às partes moles extracraniais, erosão das paredes do conduto auditivo interno e também comprometimento do ouvido médio, com destruição da cadeia ossicular e ocupação das cavidades aéreas por material hiperdenso. O diagnóstico foi compatível com otomastoidite crônica direta de provável natureza colesteomatosa.

Ao exame microscópico da secreção foi verificado a presença de ovos de Lagochilascaris minor.

Com a introdução da terapia específica com Albendazol 400mg/dia e mantida antibioticoterapia, observou-se regressão paulatina do quadro com conseqüente processo cicatricial fibrótico local.

No final do tratamento, foi feito a quantificação de linfócitos pelo método de imunofluorescência, o qual revelou valores de $1536 \mathrm{~mm}^{3}(48 \%)$ e $928 \mathrm{~mm}^{3}(29 \%)$ de CD e CD respectivamente. Com melhora clínica importante (regressão da febre, ganho de peso, recuperação do apetite e boa disposição) o paciente recebeu alta, sendo orientado retorno após 2 meses.

\section{DISCUSSÃO}

Baseado na hipótese de Smith et al, $1983^{38} \mathrm{e}$ no ciclo evolutivo experimental do parasito realizado por Campos et al, $1992^{6}$, admite-se a possibilidade do mecanismo de infecção humana ser através da ingestão da carne de animais silvestres contendo larvas encistadas na musculatura. Esta hipótese ainda é corroborada pelo fato de que a maioria dos pacientes é procedente de zona rural e tem como hábito alimentar a ingestão de carne de animais silvestres como: Dasyprocta agouti (cutia), Agouti paca (paca), Tapirus terrestris (anta), Hydrochoerus hydrochoeris (capivara) e Mazama americana (veado). Paçô et al $1991^{30}, 1992^{31}, 1993^{32}$ observaram que experimentalmente o preá e a cutia após infecção experimental com ovos do parasito servem como fonte de infecção para um carnívoro (gato doméstico). O paciente em questão é procedente de zona rural e confirmou o hábito de alimentar-se de carne de alguns destes animais silvestres.

A quantificação de linfócitos demonstrou valores dentro dos parametros normais, porém para uma avaliação adequada do estado imunológico do paciente sugerimos a execução do exame complementar no início e no final do tratamento.
Um dos fatores que contribui fortemente para dificultar a terapêutica dos pacientes infectados é o encontro de todas as fases evolutivas simultaneamente nas lesões. Durante a reprodução experimental do $L$. minor também tem se observado a presença de todas as fases evolutivas do parasito no local do abscesso ${ }^{39}$. A realização de ensaios terapêuticos com diferentes drogas, envolvendo hospedeiros experimentalmente infectados, sobre fases evolutivas específicas do parasito tem contribuído para auxiliar uma terapêutica eficaz ${ }^{43}$.

Vários anti-helmínticos já foram utilizados visando o tratamento da lagochilascariose humana, como, dietilcarbamazina, tiabendazol, mebendazol, levamisol, cambendazol e albendazol. Entretanto, insucessos têm sido registrados com a maioria dos esquemas terapêuticos empregados. Há relatos de recidivas, após um período inicial de cura aparente 2481014192225272835 .

Com respaldo na literatura 274142 utilizou-se o albendazol na dosagem de $400 \mathrm{mg} /$ dia durante 30 dias. $O$ paciente apresentou cura clínica e para posterior avaliação parasitológica foi orientado retorno após dois meses. 


\section{REFERÊNCIAS BIBLIOGRÁFICAS}

1. Artigas PT, Araujo, P, Romiti N, Ruivo M. Sobre um caso de parasitismo humano por Lagochilascaris minor Leiper, 1909, no Estado de São Paulo, Brasil. Revista do Instituto de Medicina Tropical de São Paulo 10:78-83, 1968.

2. Baracat DA, Freire EL, Aquino JL. Oto-mastoidite crônica por Lagochilascaris minor com comprometimento da região temporo-parieto-occipital. Revista da Universidade Federal do Mato Grosso 2:9-14, 1984.

3. Barbosa CAL. Avaliação da eficácia do ivermectin sobre fases do ciclo evolutivo experimtal de Lagochilascaris minor Leiper, 1909. Dissertação de Mestrado, Universidade Federal de Goiás, Goiânia, 1996.

4. Borgo AV, Andrade ALS, Pedrosa RB, Barbosa W, Komma MD. Infecção por Lagochilascaris minor - apresentação de caso. In: Resumos do XIV Congresso Da Sociedade Brasileira de Medicina Tropical e X Congresso da Sociedade Brasileira de Parasitologia, v. 3, João Pessoa, p. 391, 1978.

5. Botero D, Little MD. Two cases of human Lagochilascaris infection in Colombia. The American Journal of Tropical Medicine and Hygiene 33:381-6, 1984.

6. Campos DMB, Freire Filha LG, Vieira MA, Paçô JM, Maia MA. Experimental life cycle of Lagochilascaris minor Leiper Leiper 1909. Revista do Instituto de Medicina Tropical de São Paulo 34:277-287, 1992.

7. Campos DMB, Komma MD, Santos MAQ, Pitaluga WMNV. Lagochilascaris minor Leiper, 1909: casos diagnosticados no Departamento de Parasitologia. In: Resumos do VI Congresso da Federacion LatinoAmericana de Parasitólogos e VIII Congresso da Sociedade Brasileira de Parasitologia, São Paulo, p. 100, 1983.

8. Campos DMB, Maia MA, Freire Filha LG, Vieira MA, Carvalho SMD. Infecção por Lagochilascaris minor. Registro de um novo caso e ilações de natureza epidemiológica. Revista do Instituto de Medicina Tropical de São Paulo 33(supl):S41, 1991.

9. Campos, DMB, Paçô, JM, Barbosa, CAL. Ocorrência do ciclo auto-infectante na lagochilascariose felina experimental. Revista Brasileira de Parasitologia Veterinária. XIII Congresso Brasileiro de Parasitologia 2 (supl 1):2, 1993.

10. Campos DMB, Zanini LA, Santos ER, Paçô JM, Vieira MA. Observações parasitológicas referentes à infecção crônica por Lagochilascaris minor: resistência à Dietilcarbamazina, Levamisol, Albendazol e Ivermectin. In: Resumos do XXI Congresso da Sociedade Brasileira de Medicina Tropical, São Paulo, p.21, 1995.

11. Campos R, Vieira Bressan MCR, Little MD, Rosemberg S, Pereira VC, Masuda Z. Encefalopatia aguda por Lagochilascaris minor Leiper 1909. II Aspectos parasitológicos. In: Resumos do XXI Congresso da
Sociedade Brasileira de Medicina Tropical, São Paulo, p.74, 1985.

12. Corrêa MOA, Hyakutake S, Brandi AJ, Monteiro CG. Novo caso de parasitismo humano por Lagochilascaris minor Leiper, 1909. Revista do Instituto Adolfo Lutz 38:59-65, 1978.

13. Costa HMA, Silva AVM, Costa PR, Assis SB. Lagochilascaris minor Leiper, 1909, (Nematoda Ascaridae) de origem humana. Revista do Instituto de Medicina Tropical de São Paulo 28:126-130, 1986.

14. Draper JW, Buckley JJC. Lagochilascaris minor Leiper, 1909, from a patient in Tobago. Transactions of the Royal Society of Tropical Medicine and Hygiene 57:7, 1963.

15. Eulálio KD, Salmito MA, Lima FGC, Leal MJS. Relato de quatro casos de Lagochilascaríase. Revista da Sociedade Brasileira de Medicina Tropical 27(supl 1):319, março, 1994.

16. Fraiha $H$, Rocha MP, Araújo OJ, Barros VLRS, Primo A, Moraes MAP, Conceição JR, Oliveira JE. Patologia amazônia exótica. II: Infecção humana por Lagochilascaris minor Leiper, 1906 (Nematoda, Ascarididae). Registro de três novos casos, e formulação de nova hipótese para o mecanismo de infecção. In: Resumos do VIII Congreso da Sociedade Brasileira de Parasitologia e VI Congresso da Federación LatinoAmericana de Parasitólogos, São Paulo, p. 146, 1983.

17. Fraiha $H$, Leão RNQ, Barros VLRS, Carvalho RA. Lagochilascaríase. In: Instituto Evandro Chagas, 50 Anos de Contribuição às Ciências Biológicas e à Medicina Tropical. Belém, Fundação SESP, v. I, p. 221-242, 1986.

18. Fraiha $H$, Leão RNQ, Costa FSA. Lagochilascaríase humana e dos animais domésticos. Zoonose. Revista Internacional 1:25-33, 1989.

19. Leão RNQ, Leão Filho J, Dias LB, Calheiros LB. Infecção humana pelo Lagochilascaris minor Leiper, 1909. Registro de um caso observado no Estado do Pará (Brasil). Revista do Instituto de Medicina Tropical de São Paulo 20:300-306, 1978.

20. Leiper RT. A new nematode worm from Trinidad, Lagochilascaris minor. Proceedings Zoology Societ London 4:742-743, 1909.

21. Monteiro MR, Albuquerque HPC, Souza JM, Abdon NP, Frahia Neto H, Souza JR, Almeida AJL. Comprometimento do sacro na lagoquilascaríase. In: XXIV Congresso da Sociedade Brasileira de Medicina Tropical, Manaus, p. 98, 1988.

22. Moraes MAP, Arnaud MVC, Lima PE. Novos casos de infecção humana por Lagochilascaris minor Leiper, 1909, encontrados no Estado do Pará, Brasil. Revista do Instituto de Medicina Tropical de São Paulo 25:139-146, 1983.

23. Moraes MAP, Arnaud MVC, Macedo RC, Anglada AE. Infecção pulmonar fatal por Lagochilascaris $s p$, 
provavelmente Lagochilascaris minor Leiper, 1909. Revista do Instituto de Medicina Tropical de São Paulo 27:46-52, 1985

24. Obeid JN, Fraiha Neto H, Vieira FP, Abreu EP. Lagochilascaríase com envolvimento cerebelar. In: XXI Congresso da Sociedade Brasileira de Medicina Tropical, São Paulo, p. 80, 1985.

25. Oostburg BFJ, Varma AAO. Lagochilascaris minor infection in Surinam. Report of a case. The American Journal of Tropical Medicine and Hygiene 17:548-550, 1968.

26. Oostburg BFJ. Thiabendazole therapy of Lagochilascaris minor infection in Surinam. Report of a case. The American Journal of Tropical Medicine and Hygiene 20:580-583, 1971.

27. Oostburg BFJ. The sixth case of Lagochilascaris minor in Surinam. Tropical and Geographic Medicine p.154-159, 1992.

28. Orihuela R, Botto C, Delgado O, Ortiz A, Suarez JA, Arguello $C$. Lagochilascaríasis humana en Venezuela: descripción de un caso fatal. Revista da Sociedade Brasileira de Medicina Tropical 20:217-221, 1987.

29. Paçô JM, Campos DMB. Lagochilascaris minor Leiper, 1909: nove décadas de revisão bibliográfica. Revista de Patologia Tropical 27:11-34, 1998.

30. Paçô JM, Campos DMB, Maia MA, Freire Filha LG, Vieira MA. Lagochilascaris minor Infecção experimental em Dasyprocta agouti. Revista do Instituto de Medicina Tropical 33(supl 8):S40, 1991.

31. Paçô JM, Campos DMB, Barbosa CAL. Importância do hospedeiro intermediário no ciclo evolutivo experimental de Lagochilascaris minor. In: Resumos do XXVIII Concresso da Sociedade Brasileira de Medicina Tropical, Belém p. 100, 1992.

32. Paçô JM, Campos DMB, Maia MAM, Vieira MA. Roedores de origem silvestre na lagochilascaríase experimental. In: Resumos do XXIX Congresso da Sociedade Brasileira de Medicina Tropical, Fortaleza p. 347, 1993.

33. Pawan JL. A case of infection with Lagochilascaris minor (Leiper). Annals of Tropical Medicine and Parasitology 20:201-202, 1926.

34. Pawan JL. Another case of infection with Lagochilascaris minor (Leiper). Annals of Tropical Medicine and Parasitology 21:45-46, 1927.

35. Rocha MPC, Fraiha Neto H, Barreto Netto ACP. Infecção de ouvido médio e mastóide por Lagochilascaris minor
Leiper, 1909 (Nematoda, Ascaridiae). Relato de um caso do Sul do Estado do Pará, Amazônia, Brasil. Hiléia Médica 6:3-14, 1984.

36. Rosemberg S, Lopes MBS, Masuda Z, Campos R, Bressan MCS. Fatal encephalopathy due to Lagochilascaris minor infection. The American Journal of Tropical Medicine and Hygiene 35:575-578, 1986.

37. Santos MAQ, Campos DMB, Barnabé W. Lagochilascaris minor (Leiper, 1909) em abscesso dentário em Goiânia. Revista de Patologia Tropical 16:1-6, 1987.

38. Smith JL, Bowman DD, Little MD. Life cycle and development of Lagochilascaris sprenti (Nematoda: Ascarididae) from opossums (Marsupialia: Didelphidae) in Louisiana. Journal of Parasitology 69:736-745, 1983.

39. Souza LCS, Pinto RNL, Pacheco PRG, Pereira LIA, Campos DMB. Lagochilascaris minor, relato de dois casos. Revista da Sociedade Brasileira de Medicina Tropical 19:68, 1986.

40. Telles Filho FQ, Ciola MPP, Ioshii SO, Serafini SZ, Hofmeister R, Carneiro M. Infecção por Lagochilascaris minor (Leiper, 1909): relato de um caso. Revista da Sociedade Brasileira de Medicina Tropical 20:85, 1987.

41. Vieira MA, Oliveira JA, Barbosa CAL, Campos DMB. Atividade anti-embriogênica "in vitro" do albendazol sobre ovos de Lagochilascaris minor, Leiper, 1909. Revista de Patologia Tropical 23:221-227, 1994a.

42. Vieira MA, Campos DMB, Oliveira JA, Paçô JM, Barbosa CAL. Resultados preliminares referentes à ação do albendazol na lagochilascaríase felina experimental. Revista da Sociedade Brasileira de Medicina Tropical 27(supl 1):321, 1994b.

43. Vieira MA, Oliveira JA, Barbosa CAL. Avaliação da eficácia do albendazol na lagochilascaríase murina experimental. Revista de Patologia Tropical 25:253-262, 1996.

44. Volcan GS, Ochoa FR, Medrano CE, Valera Y. Lagochilascaris minor infection in Venezuela. Report of a case. The American Journal of Tropical Medicine and Hygiene 31:1111-1113, 1982.

45. Zacariotti AV. Lagochilascaris minor Leiper, 1909: Envolvimento do Sistema Nervoso Central com comprometimento raquiano. Monografia do Curso de Residência Médica em Neurocirurgia, Universidade Federal de Goiás, Goiânia, 1996. 